\title{
Oxidative stress and schizophrenia: A comparative cross-sectional study of multiple oxidative markers in patients and their first-degree relatives
}

\author{
Eray Guler ${ }^{1}$, Ayse Kurtulmus Calis ${ }^{2}$, Ayse Zehra Gul ${ }^{1}$, Abdurrahim Kocyigit ${ }^{1}$, and Ismet \\ Kirpinar $^{1}$ \\ ${ }^{1}$ Bezmialem Vakif University \\ ${ }^{2}$ Istanbul Medeniyet Üniversitesi Tip Fakültesi
}

January 3, 2021

\begin{abstract}
Objective: Schizophrenia(SCZ) is a heritable severe mental disorder with unknown pathogenic mechanisms. It is known that oxidative stress(OS) plays a crucial role in the pathophysiology of SCZ. Our study aimed to evaluate whether there is a relationship between the severity of illness and OS biomarkers in SCZ patients and first-degree-relatives of patients(FDRs). Methods: This study included 50 adult patients with SCZ, 50 unaffected FDRs, and 50 controls. OS biomarkers, myeloperoxidase(MPO), total oxidant status(TOS), total antioxidant status(TAS), total thiol(TT), and native thiol(NT) levels were measured by different photometric methods. Disulfide(DS) and oxidative stress index(OSI) were calculated with mathematical formulas. Results: TOS, DS, and OSI levels were significantly higher, and TAS, TT, and NT levels were lower in SCZ and FDRs than controls. In SCZ group, MPO activity was higher compared to relatives and controls. Results in this study did not provide a strong correlation between Positive\&Negative Syndrome Scale(PANSS) and other biomarkers. There was just a low negative correlation between TT and PANSS in SCZ group $(\mathrm{p}=0,041, \mathrm{r}=-0,297)$. Conclusion: OS increased significantly in SCZ, and FDRs increased slightly than controls, indicating the presence of OS in the etiology of the disease. High OS may be an effective factor for SCZ in later years.
\end{abstract}

\section{Introduction}

Schizophrenia (SCZ) is a neurodevelopmental disorder that begins in late adolescence-young adulthood ${ }^{1-3}$. It is also a mental disorder characterized by hallucinations, delusions, and cognitive deficits, with an inheritance of $80 \%$ and a lifetime risk of $1 \% 4,5$. Before the onset of psychotic illness, endophenotypic markers can also manifest in healthy relatives at a higher rate compared to the general population ${ }^{6}$. Previous medical studies have shown that the function of the brain structure, neurotransmitter anomalies, genetic and environmental factors are effective in the pathogenesis of schizophrenia ${ }^{7,8}$ and contributing causes like viral infections, low vitamin D exposure in the prenatal period, smoking, social cognition cannabis use, nutrition and childhood trauma are suggested to be vulnerability factors ${ }^{9,10}$.

Among numerous efforts to describe molecular roots of SCZ, on the top of the dopamine (DA) hypothesis, activated inflammatory pathways, specific genetic polymorphisms, neurodevelopmental and postnatal risk factors have been stated ${ }^{11,12}$. DA hypothesis has been the central idea suggesting the dysfunction in dopamine neurotransmission is responsible for the symptoms seen in SCZ, and the idea has led to therapeutical interventions to target the altered dopamine metabolism ${ }^{13}$. It suggests that hyperactivity of D2 receptor in the midbrain, especially in the mesolimbic region, leads to positive symptoms while the hypofunction of D1 receptors are demonstrated in the prefrontal cortex contributes to the negative and cognitive 
symptoms ${ }^{14-17}$. In support of this, imaging studies on SCZ patients revealed dopamine dysfunction in nigrostriatal pathways ${ }^{18,19}$. Activated immune-inflammatory response in the pathogenesis of SCZ is one of the landmark, as a variety of studies introducing the modulators of this response in the biospecimens of patients, and animal models proved that triggered immune reaction by disturbances like pre-perinatal infections and other stress factors in the early period of neurodevelopment caused the lifelong increased immune response in individuals diagnosed with SCZ. Inflammatory mechanisms are mainly executed by microglial cells (macrophages of the brain), astrocytes, and pro-inflammatory cytokines ${ }^{20,21}$. The aberrant response by these cells to infections, trauma, environmental toxins, and genetic dysfunction can lead to uncontrolled inflammation and so to neurodegeneration seen in SCZ patients ${ }^{22,23}$. Smith and collaborators used a rodent maternal infection model showing that maternal activation of IL-6 during infection caused SCZ symptoms in the adult offspring ${ }^{24}$. Increasing evidence suggests that oxidative stress (OS) can be considered as the common ground of disrupting factors in the disease development process ${ }^{25}$. Oxidative-antioxidative imbalance in favor of oxidative pathways is shown in SCZ patients as the elevated oxidative markers described in blood and cerebrospinal fluid of patients as well as decreased level of antioxidant defense component glutathione (GSH) in magnetic resonance spectroscopy (MRS) studies ${ }^{26-28}$. To identify the mechanisms referring to the impact of OS on disease etiology, several studies put emphasis on the changes in the group of cells called 'parvalbumin interneurons (PVIs)' that regulate cortical excitability and synaptic plasticity ${ }^{29}$ as well as cognitive functions ${ }^{30-32}$. PVIs are the last cells that appear during the developmental window ${ }^{33}$, and these interneurons have been proved to be affected by $\mathrm{OS}^{34,35}$. Relatively slow development of these interneurons increases their vulnerability to environmental perturbations in their maturation period as an animal study puts forth repetitive activation of IL-6/Nox2 pathway in PVIs alters the redox balance on the side of pro-oxidant state could explain the delayed effects observed in SCZ models ${ }^{36,37}$. The main dysfunction of PVI cells in SCZ pathophysiology is considered hypofunction of their N-methyl-D-aspartate receptors (NMDARs) ${ }^{38}$ and proven that NMDARs are essential for maintaining the normal oxidative status, a variety of mechanisms disrupting the function of these receptors have been found to led psychotic symptoms ${ }^{39}$. Another study about NMDARs concluded that after using receptor antagonists in subclinical doses, both negative and positive symptoms of SCZ came off ${ }^{40}$.

Antioxidant defense breakdowns as a cause of oxidative damage in SCZ patients involve glutathione (GSH), superoxide dismutase (SOD), and thiol metabolisms. GSH synthesis deficits have been reported in a group of SCZ patients ${ }^{41}$. Preclinical models show that GSH depletion triggers persistent psychotic symptoms during the postnatal period ${ }^{42}$, and GSH deficit leads to NMDAR hypofunction as well as synaptic disruptions 43. Reversely, a GSH precursor drug, N-acetylcysteine (NAC) supply limits PVI impairment in psychosis animal models ${ }^{35}$. Add-on NAC treatment also proved to mitigate SCZ symptoms in humans ${ }^{44}$. There are several studies showing a relationship between lower levels of GSH and disease severity, as well as brain volume changes ${ }^{45,46}$. Recent clinical studies have found significantly lower levels of SOD and GSH along with higher levels of lipid oxidation markers in terms of oxidative imbalance by examining blood, cerebrospinal fluid (CSF), and post-mortem tissue $e^{47,48}$. Thiol-disulfide balance is another metabolic indicator of oxidative stress ${ }^{49}$, and in cases where oxidative stress increases, thiol-disulfide balance shifts in favor of disulfide. The level of thiol-containing compounds such as glutathione, cysteine, homocysteine, and $\mathrm{N}$-acetylcysteine are found altered in SCZ patient biospecimens referring to the role of oxidative stress in etiology ${ }^{28,50,51}$. Elevated homocysteine levels are reported in a variety of clinical studies, and one of them suggest the possible use of this marker as a risk factor in $\mathrm{SCZ}^{52-55}$.

Other evidence for oxidative stress impact in schizophrenia may be explained by deficits in the lipid-rich white matter, which is highly sensitive to $\mathrm{OS}^{56,57}$. Oxidative stress disrupts cellular signaling pathways, which can also contribute to pathophysiology ${ }^{58}$. DNA damage has also been implicated as a target of $\mathrm{H}_{2} \mathrm{O}_{2}$ due to its large number of negatively charged ions binding cations under oxidative stress ${ }^{59}$.

Eliciting the genetic basis of schizophrenia, several Genome-Wide Association Studies (GWAS) described multiple high-risk genes, and among them, immune regulatory genes (namely, MHC) are found to be the most reliable evidence in the genesis of the disorder ${ }^{60,61}$. Many other genes associated with SCZ were associated with an antioxidant defense, such as Dysbindin ${ }^{62}$ and the gene of GSH-synthesizing enzyme, glutamate- 
cysteine ligase modifier (GCLM) subunit ${ }^{63}$. A meta-analysis has found the entity's estimated heritability around $80 \%^{4}$. Several efforts to search for oxidative damage traces in the patient's peripheral blood described possible diagnostic markers for SCZ, and mostly strong evidence was found, but contradictory results prevented the use of markers as a routine tool.

This study examines multiple oxidative markers in the peripheral blood of schizophrenia patients and their first-degree relatives (FDR). Assessment of any correlation present between patients' OS parameter levels and the severity of illness, which is determined by PANSS total score. Investigating the oxidative parameters in both SCZ patients and their first-degree relatives (FDR), our study searched a possible endophenotypic role of FDRs of SCZ patients for the first time. Since there are only limited clinical studies, including FDRs of schizophrenia patients, the current study fills the gap in the literature by testing if OS markers are elevated in the patient's relatives, which would refer to the genetic background of the entity, also confirm the etiological role of OS.

\section{Materials and Methods}

To make the comparison of the oxidative parameters of schizophrenia patients' relatives with other groups and the investigation of the roles of the systemic OS in schizophrenia pathogenesis possible, this observational case-control study was planned. The study complies with the Declaration of Helsinki and was approved by the clinical research ethics committee of Bezmialem Vakif University $(14 / 23$ - 24.07.2019). Each volunteer has been asked to provide written informed consent. First-degree relatives and healthy controls were assessed through a semi-structured psychiatric interview and a Social Cognition Scale, which assessed the domains: emotional processing, social perception, and attributional style in a Turkish population. The schizophrenic patients had been diagnosed according to the diagnostic criteria of Diagnostic and Statistical Manual of Mental Disorders, $5^{\text {th }}$ Edition, Text Revision (DSM-V-TR), and followed for at least three years in the psychiatry clinic of Bezmialem Vakif University School of Medicine Hospital ${ }^{64}$.

From March 2017 to January 2018, this cross-sectional study included 50 adult patients (sex: 20 females - 30 males; age: 30-52) who were diagnosed with schizophrenia in Bezmialem Vakif University Medical Faculty Hospital. For the healthy control group (sex: - 19 females - 31 males; age: 27-50) and the patients' relatives group (sex: 22 females - 28 males; age: 29-49), 50 participants were included in each study group with age and sex-matched with the patient group, and no active psychopathology was detected in the psychiatric interview.

The patients with any systemic disease that might affect systemic OS parameters were excluded from the study if they met one or more of the following criteria: chronic pathological diseases, alcohol-cigarette, and substance abuse or dependence, cardiovascular diseases, diabetes mellitus, hepatic or renal failure, autoimmune diseases, active infection, active or chronic inflammatory diseases, obesity (greater than body mass index $30 \mathrm{~kg} / \mathrm{m}^{2}$ ), and treatment with anti-inflammatory, antioxidant, or immunosuppressive medications.

\section{Socio-Demographic Data Form}

Sociodemographic and clinical data forms variables such as comorbid medical diseases, alcohol/substance use, smoking status, family histories, job, education, and Body Mass Index (BMI) were recorded for all volunteers.

\section{Score of PANSS}

Psychiatrists who were blind to the clinical status and treatment conditions were employed to assess the psychopathology of the patient of schizophrenia with the PANSS on the same day of blood sampling. PANSS was developed in 1987 by Kay, Opler, and Fiszbein ${ }^{65}$. It is a semi-structured interview scale with 30 items, which are rated on a severity scale ranging from 1 to 7 . 7 out of 30 items belong to the positive syndrome subscale, 7 to the negative syndrome subscale, and the remaining 16 to the general psychopathology subscale. The total PANSS score is obtained by summing all individual subscale scores.

Blood Sample Collection 
Blood was collected after 12 hours of fasting from all volunteers from the cubital vein to gel biochemistry tubes for laboratory analysis. The blood was centrifuged at 3000xrpm for 10 minutes to separate the serum. All serums were aliquoted and stored at $-80^{\circ} \mathrm{C}$ until further biochemistry analysis.

\section{Determination of Total Oxidant Status}

Serum TOS level was measured using a fully automatic photometric method developed by Erel ${ }^{66}$. The principle of the method is based on the oxidizing character of serum oxidants to the ferrous ions of odianisidine into ferric ions. Ferric ions formed in an acidic environment as a result of this oxidation take a visible color with xylenol orange. The resulting color density is correlated with the level of oxidants in the serum. The standard of the method is $\mathrm{H}_{2} \mathrm{O}_{2}$. Results are given as $\mu \mathrm{mol} / \mathrm{L} \mathrm{H}_{2} \mathrm{O}_{2}$ equivalent. The sensitivity of the method is $2 \%$.

\section{Determination of Total Antioxidant Status}

Serum TAS level is a fully automatic colorimetric method developed by Erel ${ }^{67}$. The principle of the method is based on measuring the amount of $\mathrm{OH}^{-}$radical. The ferrous ion of o-diasidine with $\mathrm{H}_{2} \mathrm{O}_{2}$ reacts to the Fenton-type reaction to produce the $\mathrm{OH}^{-}$radical, and color change occurs due to o-diasidine. Serum antioxidants neutralize oxidants and prevent color change. This method determines the antioxidant capacity against oxidative free radical reactions initiated by $\mathrm{OH}^{-}$. The standard of this method is Trolox, which is an alpha-tocopherol analog. Results are given as mmol/L Trolox equivalent. The sensitivity of the method is 3 $\%$.

\section{Calculation of Oxidative Stress Index}

OSI value is the most important indicator of oxidative stress. The serum OSI value is calculated by dividing the TOS value by the TAS value. OSI (arbitrary unit) = TOS $\left(\mu \mathrm{mol} \mathrm{H}_{2} \mathrm{O}_{2} \mathrm{eq} / \mathrm{L}\right) / \mathrm{TAS}(\mathrm{mmol}$ Trolox eq/L).

\section{Determination of Thiol/Disulfide Homeostasis (TDH)}

New biomarkers of oxidative stress are thiol-disulfide homeostasis. Total thiol, native thiol, and disulfide levels are measured in serum with this method developed by Erel and Neselioglu ${ }^{68}$. The principle of this method is based on the reduction of dynamic disulfide bonds (-S-S-) in serum, to native thiol groups (-SH HS-) by sodium borohydride $\left(\mathrm{NaBH}_{4}\right)$. Total thiol level is measured photometrically using Ellman reagent. The disulfide level is calculated by subtracting the native thiol level from the total thiol level and taking half of it. The unit of measurement for thiol-disulfide homeostasis is $\mu \mathrm{mol} / \mathrm{L}$.

\section{Activity of Myeloperoxidase}

MPO is an enzyme related to oxidative stress. The activity of MPO was determined by the method of Bradley et al. ${ }^{69}$, which is based on kinetic measurement of the formation rate of the colorful product of the oxidation of o-dianisidine with MPO in the presence of $\mathrm{H}_{2} \mathrm{O}_{2}$, at $460 \mathrm{~nm}$. MPO activity was expressed as units per liter of serum (U/L).

\section{Statistical Analysis}

All statistical analyses were conducted with IBM SPSS version 24.0 software (IBM Corporation, Armonk, NY, USA). All data are given as mean \pm standard deviation. The distributions of the data were found with a single sample Kolmogorov-Smirnov test, and it was found to be a normal distribution. One-way analysis of variance (ANOVA) was used to determine whether there was a statistically significant difference between the groups. Pearson correlation test was performed to evaluate the correlation between variables. A p-value less than or equal to 0.05 was accepted as statistically significant.

\section{Results}

\section{Socio-Demographic Data}

A total of 50 patients with schizophrenia, 50 relatives of schizophrenia patients, and 50 healthy controls have been listed. The socio-demographic variables of the study populations are presented in Table 1 . There 
were no significant age, sex, smoking, and BMI differences between the schizophrenia, relative, and control groups $(\mathrm{p}>0,05)$. The average of education level was $11.10 \pm 5.39$ in the control group, $9.81 \pm 4.82$ in the relative group, and $7.76 \pm 3.98$ in the SCZ group $(\mathrm{p}<0.05)$. Marital status was found in $82 \%$ of the patients, $48 \%$ of the relatives, without statistical differences between the two groups, and in $42 \%$ of the control group $(\mathrm{p}<0.01)$. Job Status of the SCZ group has been observed at the lowest rate among the three groups $(\mathrm{p}<0.01)$ (Table 1).

\section{Table 1}

\section{Correlation Analysis of PANSS and OS}

Correlation between OS biomarkers and total PANSS was shown in the patient group. Results in this study did not provide a strong correlation between PANSS and other oxidative stress biomarkers. There was just a low negative correlation between TT and total PANSS in the patient group $(\mathrm{r}=-0,703, \mathrm{p}=0,041)$.

\section{Oxidative Stress Status}

In our current study, we found that serum TOS and OSI levels have been found significantly higher $(p$ $<0,01)$, and TAS levels were lower in the SCZ group than in the relatives and control groups $(p<0,01)$. The relatives' group's serum TOS and OSI levels were higher than the control group, and TAS levels were lower in the relatives' group than the control group. As shown in the figure, in the SCZ group, MPO enzyme activity tended to be higher compared to relatives and control groups $(p<0,01)$ (Figure 1).

\section{Figure 1}

\section{Thiol-Disulfide Homeostasis}

Figure 2 reports thiol-disulfide homeostasis of SCZ, relatives, and healthy controls. It has been found that plasma total and native thiol levels were highest in the controls, relatively lower in relatives, and lowest in the patients. There was a statistically significant difference in total and native thiol between the three groups $(\mathrm{p}<0,05)$. Plasma thiol-disulfide levels were highest in the SCZ group, as shown in figure 2. The thiol-disulfide level was relatively lower in the relatives' group and was lowest in the control group. There was a statistically significant difference in thiol-disulfide levels between the three groups $(\mathrm{p}<0,05)$ (Figure 2).

Figure 2

\section{Discussion}

Like most psychiatric disorders, SCZ is not a single entity, multiple factors are involved in the pathogenesis; specific genetic polymorphisms, neurodevelopmental and postnatal risk factors, and environmental influences. SCZ has a clinical heterogeneity in nature, which should be regarded in human studies. However, growing knowledge in the relationship between SCZ and immune system perturbations (and so oxidative imbalance) has led many researchers to put their efforts into this issue. Among loads of mechanisms suggested for etiology, OS has been considered as a final common pathway that into many mechanisms merge.

With this present study, we support the idea that OS markers might serve as a useful tool for diagnosis and place new targets for intervention. Our findings refer to the causal association of OS in SCZ progress by displaying significantly higher levels of oxidative parameters in the blood samples of the patients compared to other groups. Higher levels of markers found in the study included the plasma TOS and OSI levels with MPO enzyme activity. Whereas patients had lower levels of TAS and lowest of plasma total and native thiol. Similar studies investigating inflammatory parameters in SCZ patients defined both diagnostic and theranostic markers ${ }^{70-73}$, and findings in a group of patients have led to the term called 'high inflammatory biotype $^{74}$. Another meta-analysis examining oxidative markers has identified two groups of the indicator; one is 'state markers' such as total antioxidant status, plasma nitrite, red blood cell catalase, while the other group is 'trait markers' including red blood cell catalase ${ }^{75}$. However, some contradictory results show that using oxidative status as a diagnostic clue for high-risk people and a disease marker for patients have not 
proved to be reliable in terms of the insignificant difference of parameters ${ }^{76,77}$. This heterogeneity among results may be due to the different immunological, biochemical, endophenotypic profiles of individuals, and identifying the high-risk subgroups in specific stages of the entity would help us get more clear results.

More importantly, our recent work is unique for contributing to data by showing the average levels of markers found in first-degree relatives' blood samples while indicating the genetic background of the syndrome. For the whole parameters explained before, FDR blood samples displayed mid-levels in between patients and controls, except for the thiol-disulfide levels. This fact imposes questions like how we can benefit from relatives to identify some risky endophenotypes and how these phenotypes turn up to the clinical presentation stage, by which mechanisms, as well as how the gene by environmental interactions occur. Based on the findings of the research, the increased prevalence of autoimmune diseases in patients' relatives besides blood cytokine changes may imply that immune abnormalities could predispose to psychosis, and it may help to categorize immune endophenotypes of $\mathrm{SCZ}^{78}$. Moreover, it has been shown that by measuring the high clinical risk for psychosis (CHR) in individuals and then combining it with offered endophenotypes, psychosis progression might be predicted and intervened before its onset ${ }^{79}$. Finally, a review gathering of various studies highlights that oxidative status may help to identify specific endophenotypes of individuals at risk ${ }^{80}$, and with this current research, we support this hypothesis.

Because of the bias involving immunological studies, investigating the causal role of inflammation-OS in SCZ pathophysiology requires attention. This kind of studies are open to interference from various factors, including psychological stress, medication, and sleep. The fact withdraws us to make strong assumptions about the temporal relationship between inflammation (and therefore OS) and the onset of the entity ${ }^{81}$. However, treatment studies may provide valuable insights looking into disease pathophysiology, and trials implicate that some patients with immune imbalances benefit from adjunct therapies such as anti-inflammatories; celecoxib and minocycline showed improvements in both negative symptoms and cognitive function. The benefits of antioxidants may point to the etiological role of oxidative imbalance in a subgroup of patients with SCZ, and positive results hold promise for clinical use soon. Immunotherapy, on the other hand, especially the monoclonal antibody treatments, seems to be effective in some patients with immune disturbances ${ }^{82}$, but more comprehensive randomized clinical trials are required to validate the efficacy of these therapies.

With this present study, we support the idea that OS markers might serve as a useful tool for risk estimation and diagnosis as well as placing new targets for intervention in schizophrenia patients. Our findings refer to the causal role of OS in disease progression by displaying significantly higher levels of oxidative markers in the bloods of patients while the marker levels of first-degree relatives (FDR) in between patients and controls. The unique data of average multiple oxidative marker levels found in first-degree relatives' samples contribute to the literature by indicating the genetic background of the syndrome. Higher levels of markers found in patient samples included the plasma total oxidant status (TOS) and oxidative stress index (OSI) levels with myeloperoxidase (MPO) enzyme activity. Patients had significantly lower levels of total antioxidant status (TAS) and had the lowest level of plasma total and native thiol. FDR blood samples displayed mid-levels in between patients and controls for the whole marker profile (TOS, OSI, MPO, TAS, TT, and NT) except for the thiol-disulfide levels.

Growing knowledge in the relationship between SCZ and oxidative imbalance has led researchers to investigate if oxidative stress plays a role in the etiology as a causal factor or it is an outcome of a pathological process ${ }^{83}$. Various studies investigating oxidant-antioxidant biomarkers in drug-naive first-episode psychosis (FEP) patients showed an increased pro-oxidative status (higher levels of homocysteine, IL-6, and TNF- $\alpha$ and lower levels of TAS, docosahexaenoic acid (DHA) in patients compared to healthy controls (Oxidative Stress and Inflammation in First-Episode Psychosis: A Systematic Review and Meta-analysis). One study found significantly lower antioxidant marker paraoxonase 1 (PON1) activity with increased TRAP values in FEP patients and showed antioxidant effects of antipsychotic drug risperidone ${ }^{84}$. Most of the drug studies focused on antipsychotics when investigating the effects of these drugs on oxidative status (Fond vd., 2015), while some suggested the use of antioxidant agents like N-acetylcysteine (NAC) as a treatment option in the early stages of the psychotic condition ${ }^{85,86}$. Therefore, the idea of using redox-state biomarkers in risk 
prediction and monitoring treatment response might open new doors in SCZ research. A study contradicting to these data found no difference before-after treatment with antipsychotics regarding oxidative status but proved higher levels of oxidant markers in patients plasma (malondialdehyde (MDA), apo B-basal MDA, and red blood cell superoxide dismutase activity) with lower serum arylesterase and whole blood- glutathione peroxidase $(\mathrm{GPx})$ activities in the FEP group ${ }^{87}$. Recent reports also support the causal role of OS in psychosis development by displaying oxidative markers associated with early stages of the disease ${ }^{88}$, D-amino acid oxidase activator predicts the progression of prodromal syndromes to first-episode psychosis, similarities in serum oxidative stress markers and inflammatory cytokines in patients with overt schizophrenia at early and late stages of chronicity. Our current results are consistent with a meta-analysis reporting lower TAS levels (Meta-analysis of oxidative stress in schizophrenia) and additionally show a significant decrease in both total and native thiol plasma levels in patients compared to controls. The meta-analysis has identified two marker groups as an indicator for disease progression ; one is 'state markers' such as total antioxidant status, plasma nitrite, red blood cell catalase and the other group is ' trait markers' including red blood cell catalase $^{47}$. It has been shown that by measuring the high clinical risk for psychosis (CHR) in individuals and then combining it with determined endophenotypes, psychosis progression might be predicted and intervened before its onset ${ }^{39}$. Further, a review gathering various studies highlights that oxidative status may help to identify specific endophenotypes of individuals at risk ${ }^{89}$. Our research showing the mid-level oxidative markers in patient relatives' blood supports the idea of risk prediction can be made before the onset of disease in individuals with higher oxidative parameters. This current finding imposes questions like how we can benefit from patient relatives to identify some risky endophenotypes and how these phenotypes turn up to clinical presentation stage, by which mechanisms, as well as how the gene by environmental interactions occur.

Studies involving first-degree relatives of schizophrenia patients have identified the increased oxidative stress and inflammation in these individuals ${ }^{90,91}$. Our study contributes to this data with raised levels of multiple oxidative parameters in FDRs compared to healthy controls. Still, an evidence belonging to irregular concentrations of the trait markers for SCZ apart from state ones, is needed to fill the gap in the literature, including relatives of patients.

Some contradictory results show that using oxidative status as a diagnostic clue identifying high-risk people, and a disease marker have not proved to be reliable, various studies found an insignificant difference for oxidative parameters between patients and controls $^{27,46}$. A study claimed that paraoxonase activity, together with oxidative-nitrosative stress (O\&NS) biomarkers are not good indicators for chronic SCZ by showing no association between patients and controls ${ }^{11}$. This heterogeneity among results may be due to the different immunological, biochemical, endophenotypic profiles of individuals, and the clinical heterogeneity of SCZ disease. Studies involving OS are open to interference from various factors, including psychological stress, comorbid diseases, medication, and sleep as in immunological ones. Thus, investigating the causal role of OS in SCZ pathophysiology requires great attention, and this fact withdraws us to make strong assumptions about the temporal relationship between inflammation (and therefore OS) and the onset of the entity ${ }^{92}$.

Our clinical study contributes to literature comparing three groups (patients, their unaffected first-degree relatives, healthy controls) regarding individuals' oxidative status. It reports significant difference or multiple oxidative markers in between these groups. The unique finding of this work, patient relatives' mid-level values of oxidative markers, suggests that oxidative damage observed in individuals may result from a genetic vulnerability for SCZ. Taken together, this study enhances the oxidative stress research in SCZ and calls for future investigations to fill the gaps in the issue. Current findings of our work need to be replicated with larger, well-designed studies excluding all intervening factors, and related socio-demographic and clinical variables should be analyzed before offering a true association between OS and SCZ.

\section{Conclusions}

The involvement of oxidative stress in SCZ pathophysiology is a widely accepted concept due to numerous findings on patient samples like peripheral blood, CNS, and post-mortem tissues. Still, which mechanisms lead to oxidative damage remains unclear, and arduous interdisciplinary work is required to enlighten the 
steps of disease progression. Our study contributes to the literature by analyzing multiple oxidative parameters in peripheral blood samples of both patients, and their unaffected first-degree relatives and claims that high levels of oxidative biomarkers would possibly be the indicator of SCZ progression in healthy individuals.

\section{Keypoint}

- There is high oxidative stress and low antioxidant capacity in schizophrenia and relatives.

- If the family has a history of schizophrenia, high oxidative stress levels can be a risk factor.

- Reduction of oxidative stress shall be taken into consideration when treatment plans for schizophrenia and relatives are being made.

\section{Declaration Of Interest Statement}

No potential conflict of interest was reported by the authors

\section{Funding details}

This research did not receive any special grant from public funding agencies, commercial or nonprofit sectors.

\section{References}

1. Murray RM, Lewis SW. Is schizophrenia a neurodevelopmental disorder? British medical journal (Clinical research ed).1987;295(6600):681.

2. Weinberger DR. Implications of normal brain development for the pathogenesis of schizophrenia. Archives of general psychiatry.1987;44(7):660-669.

3. Van Os J, Kenis G, Rutten BP. The environment and schizophrenia.Nature. 2010;468(7321):203-212.

4. Sullivan PF, Kendler KS, Neale MC. Schizophrenia as a complex trait: evidence from a meta-analysis of twin studies. Archives of general psychiatry. 2003;60(12):1187-1192.

5. James SL, Abate D, Abate KH, et al. Global, regional, and national incidence, prevalence, and years lived with disability for 354 diseases and injuries for 195 countries and territories, 1990-2017: a systematic analysis for the Global Burden of Disease Study 2017. The Lancet.2018;392(10159):1789-1858.

6. Bitanihirwe BK, Woo T-UW. Oxidative stress in schizophrenia: an integrated approach. Neuroscience $\mathcal{G}^{3}$ Biobehavioral Reviews.2011;35(3):878-893.

7. Jones AL, Mowry BJ, Pender MP, Greer JM. Immune dysregulation and self-reactivity in schizophrenia: Do some cases of schizophrenia have an autoimmune basis? Immunology and cell biology. 2005;83(1):9-17.

8. Loehrs L, Hasan A. Risk factors for the development of schizophrenia.Fortschritte der NeurologiePsychiatrie. 2019;87(2):133-143.

9. Caldani S, Vialatte F-B, Baelde A, et al. Classification of vulnerability levels using multivariate biomarkers in schizophrenia: a machine-learning approach. 2020.

10. $<10.3892 @$ or.2018.6180.pdf $>$.

11. Boll KM, Noto C, Bonifácio KL, et al. Oxidative and nitrosative stress biomarkers in chronic schizophrenia. Psychiatry research.2017;253:43-48.

12. Alam R, Abdolmaleky HM, Zhou JR. Microbiome, inflammation, epigenetic alterations, and mental diseases. American Journal of Medical Genetics Part B: Neuropsychiatric Genetics.2017;174(6):651-660.

13. Reynolds GP. Receptor mechanisms in the treatment of schizophrenia.Journal of psychopharmacology. 2004;18(3):340-345.

14. Coward D, Dixon K, Enz A, et al. Partial brain dopamine D2 receptor agonists in the treatment of schizophrenia. Psychopharmacology Bulletin. 1989;25(3):393-397. 
15. Kapur S. Psychosis as a state of aberrant salience: a framework linking biology, phenomenology, and pharmacology in schizophrenia. American journal of Psychiatry. 2003;160(1):13-23.

16. Toda M, Abi-Dargham A. Dopamine hypothesis of schizophrenia: making sense of it all. Current psychiatry reports. 2007;9(4):329-336.

17. Davis KL, Kahn RS, Ko G, Davidson M. Dopamine in schizophrenia: a review and reconceptualization. The American journal of psychiatry. 1991.

18. McCutcheon RA, Abi-Dargham A, Howes OD. Schizophrenia, dopamine and the striatum: from biology to symptoms. Trends in neurosciences.2019;42(3):205-220.

19. Birtwistle J, Baldwin D. Role of dopamine in schizophrenia and Parkinson's disease. British journal of nursing.1998;7(14):832-841.

20. Cunningham C, Wilcockson DC, Campion S, Lunnon K, Perry VH. Central and systemic endotoxin challenges exacerbate the local inflammatory response and increase neuronal death during chronic neurodegeneration. Journal of Neuroscience. 2005;25(40):9275-9284.

21. Dantzer R. Cytokine-induced sickness behavior: where do we stand?Brain, behavior, and immunity. $2001 ; 15(1): 7-24$.

22. Pérez-Neri I, Ramírez-Bermúdez J, Montes S, Ríos C. Possible mechanisms of neurodegeneration in schizophrenia. Neurochemical research. 2006;31(10):1279-1294.

23. Sparkman NL, Johnson RW. Neuroinflammation associated with aging sensitizes the brain to the effects of infection or stress.Neuroimmunomodulation. 2008;15(4-6):323-330.

24. Smith SE, Li J, Garbett K, Mirnics K, Patterson PH. Maternal immune activation alters fetal brain development through interleukin-6. Journal of Neuroscience. 2007;27(40):10695-10702.

25. Emiliani FE, Sedlak TW, Sawa A. Oxidative stress and schizophrenia: recent breakthroughs from an old story. Current opinion in psychiatry. 2014;27(3):185.

26. Gillard JH, Waldman AD, Barker PB. Clinical MR neuroimaging: physiological and functional techniques. Cambridge University Press; 2010.

27. Zhang M, Zhao Z, He L, Wan C. A meta-analysis of oxidative stress markers in schizophrenia. Science China Life Sciences.2010;53(1):112-124.

28. Do K, Trabesinger A, Kirsten-Kruger M, et al. Schizophrenia: glutathione deficit in cerebrospinal fluid and prefrontal cortex in vivo. European Journal of Neuroscience. 2000;12(10):3721-3728.

29. Nakazawa K, Zsiros V, Jiang Z, et al. GABAergic interneuron origin of schizophrenia pathophysiology. Neuropharmacology.2012;62(3):1574-1583.

30. Inan M, Petros TJ, Anderson SA. Losing your inhibition: linking cortical GABAergic interneurons to schizophrenia. Neurobiology of disease. 2013;53:36-48.

31. Lodge DJ, Behrens MM, Grace AA. A loss of parvalbumin-containing interneurons is associated with diminished oscillatory activity in an animal model of schizophrenia. Journal of Neuroscience.2009;29(8):23442354 .

32. Uhlhaas PJ, Singer W. Abnormal neural oscillations and synchrony in schizophrenia. Nature reviews neuroscience. 2010;11(2):100-113.

33. Sullivan EM, O'Donnell P. Inhibitory interneurons, oxidative stress, and schizophrenia. Schizophrenia bulletin. 2012;38(3):373-376. 
34. Johnson AW, Jaaro-Peled H, Shahani N, et al. Cognitive and motivational deficits together with prefrontal oxidative stress in a mouse model for neuropsychiatric illness. Proceedings of the National Academy of Sciences. 2013;110(30):12462-12467.

35. Cabungcal J-H, Counotte DS, Lewis EM, et al. Juvenile antioxidant treatment prevents adult deficits in a developmental model of schizophrenia. Neuron. 2014;83(5):1073-1084.

36. Behrens MM, Sejnowski TJ. Does schizophrenia arise from oxidative dysregulation of parvalbumininterneurons in the developing cortex? Neuropharmacology. 2009;57(3):193-200.

37. Behrens MM, Ali SS, Dao DN, et al. Ketamine-induced loss of phenotype of fast-spiking interneurons is mediated by NADPH-oxidase.Science. 2007;318(5856):1645-1647.

38. Hardingham GE, Do KQ. Linking early-life NMDAR hypofunction and oxidative stress in schizophrenia pathogenesis. Nature Reviews Neuroscience. 2016;17(2):125.

39. Barron H, Hafizi S, Andreazza AC, Mizrahi R. Neuroinflammation and oxidative stress in psychosis and psychosis risk. International journal of molecular sciences. 2017;18(3):651.

40. Krystal JH, Karper LP, Seibyl JP, et al. Subanesthetic effects of the noncompetitive NMDA antagonist, ketamine, in humans: psychotomimetic, perceptual, cognitive, and neuroendocrine responses.Archives of general psychiatry. 1994;51(3):199-214.

41. Juckel G, Manitz MP, Brune M, Friebe A, Heneka MT, Wolf RJ. Microglial activation in a neuroinflammational animal model of schizophrenia - a pilot study. Schizophrenia research.2011;131(1-3):96-100.

42. Jacobsen J, Rodriguiz R, Mork A, Wetsel W. Monoaminergic dysregulation in glutathione-deficient mice: possible relevance to schizophrenia? Neuroscience. 2005;132(4):1055-1072.

43. Steullet P, Neijt H, Cuenod M, Do K. Synaptic plasticity impairment and hypofunction of NMDA receptors induced by glutathione deficit: relevance to schizophrenia. Neuroscience. 2006;137(3):807-819.

44. Lavoie S, Murray MM, Deppen P, et al. Glutathione precursor, N-acetyl-cysteine, improves mismatch negativity in schizophrenia patients. Neuropsychopharmacology. 2008;33(9):2187-2199.

45. Yao JK, Reddy RD, van Kammen DP. Human plasma glutathione peroxidase and symptom severity in schizophrenia. Biological psychiatry.1999;45(11):1512-1515.

46. Fraguas D, Gonzalez-Pinto A, Mico JA, et al. Decreased glutathione levels predict loss of brain volume in children and adolescents with first-episode psychosis in a two-year longitudinal study.Schizophrenia research. 2012;137(1-3):58-65.

47. Flatow J, Buckley P, Miller BJ. Meta-analysis of oxidative stress in schizophrenia. Biological psychiatry. 2013;74(6):400-409.

48. Gawryluk JW, Wang J-F, Andreazza AC, Shao L, Young LT. Decreased levels of glutathione, the major brain antioxidant, in post-mortem prefrontal cortex from patients with psychiatric disorders.International Journal of Neuropsychopharmacology.2011;14(1):123-130.

49. McBean GJ. Cysteine, glutathione, and thiol redox balance in astrocytes. Antioxidants. 2017;6(3):62.

50. Gysin R, Kraftsik R, Boulat O, et al. Genetic dysregulation of glutathione synthesis predicts alteration of plasma thiol redox status in schizophrenia. Antioxidants $\mathscr{E}$ redox signaling.2011;15(7):2003-2010.

51. Berk M, Copolov D, Dean O, et al. N-acetyl cysteine as a glutathione precursor for schizophrenia - a double-blind, randomized, placebo-controlled trial. Biological psychiatry.2008;64(5):361-368.

52. Susser E, Brown AS, Klonowski E, Allen RH, Lindenbaum J. Schizophrenia and impaired homocysteine metabolism: a possible association. Biological Psychiatry. 1998;44(2):141-143. 
53. Levine J, Stahl Z, Sela BA, Gavendo S, Ruderman V, Belmaker RH. Elevated homocysteine levels in young male patients with schizophrenia.American Journal of Psychiatry. 2002;159(10):1790-1792.

54. Muntjewerff J-W, Kahn RS, Blom HJ, den Heijer M. Homocysteine, methylenetetrahydrofolate reductase and risk of schizophrenia: a meta-analysis. Molecular psychiatry. 2006;11(2):143-149.

55. Brown AS, Bottiglieri T, Schaefer CA, et al. Elevated prenatal homocysteine levels as a risk factor for schizophrenia. Archives of general psychiatry. 2007;64(1):31-39.

56. Konat GW, Wiggins RC. Effect of reactive oxygen species on myelin membrane proteins. Journal of neurochemistry.1985;45(4):1113-1118.

57. Takahashi N, Sakurai T, Davis KL, Buxbaum JD. Linking oligodendrocyte and myelin dysfunction to neurocircuitry abnormalities in schizophrenia. Progress in neurobiology. 2011;93(1):13-24.

58. Tristan C, Shahani N, Sedlak TW, Sawa A. The diverse functions of GAPDH: views from different subcellular compartments. Cellular signalling. 2011;23(2):317-323.

59. Sharpe MA, Robb SJ, Clark JB. Nitric oxide and Fenton/Haber-Weiss chemistry: nitric oxide is a potent antioxidant at physiological concentrations. Journal of neurochemistry. 2003;87(2):386-394.

60. Jia P, Wang L, Meltzer HY, Zhao Z. Common variants conferring risk of schizophrenia: a pathway analysis of GWAS data. Schizophrenia research. 2010;122(1-3):38-42.

61. Sekar A, Bialas AR, de Rivera H, et al. Schizophrenia risk from complex variation of complement component 4. Nature.2016;530(7589):177-183.

62. Wang $\mathrm{H}, \mathrm{Xu}$ J, Lazarovici P, Zheng W. Dysbindin-1 involvement in the etiology of schizophrenia. International journal of molecular sciences. 2017;18(10):2044.

63. Tosic M, Ott J, Barral S, et al. Schizophrenia and oxidative stress: glutamate cysteine ligase modifier as a susceptibility gene. The American Journal of Human Genetics. 2006;79(3):586-592.

64. First MB, Pincus HA. The DSM-IV Text Revision: rationale and potential impact on clinical practice. Psychiatric services.2002;53(3):288-292.

65. Kay SR, Fiszbein A, Opler LAJSb. The positive and negative syndrome scale (PANSS) for schizophrenia. 1987;13(2):261-276.

66. Erel OJCb. A new automated colorimetric method for measuring total oxidant status. 2005;38(12):11031111.

67. Erel OJCb. A novel automated method to measure total antioxidant response against potent free radical reactions. 2004;37(2):112-119.

68. Erel O, Neselioglu SJCb. A novel and automated assay for thiol/disulphide homeostasis. 2014;47(18):326332.

69. Bradley PP, Priebat DA, Christensen RD, Rothstein GJJoID. Measurement of cutaneous inflammation: estimation of neutrophil content with an enzyme marker. 1982;78(3):206-209.

70. Mori N, McEvoy JP, Miller BJJSr. Total and differential white blood cell counts, inflammatory markers, adipokines, and the metabolic syndrome in phase 1 of the clinical antipsychotic trials of intervention effectiveness study. 2015;169(1-3):30-35.

71. Nielsen PR, Agerbo E, Skogstrand K, Hougaard DM, Meyer U, Mortensen PBJBp. Neonatal levels of inflammatory markers and later risk of schizophrenia. 2015;77(6):548-555.

72. Goldsmith D, Rapaport M, Miller BJMp. A meta-analysis of blood cytokine network alterations in psychiatric patients: comparisons between schizophrenia, bipolar disorder and depression. 2016;21(12):1696. 
73. Lai C-Y, Scarr E, Udawela M, Everall I, Chen WJ, Dean BJWjop. Biomarkers in schizophrenia: a focus on blood based diagnostics and theranostics. 2016;6(1):102.

74. Boerrigter D, Weickert TW, Lenroot R, et al. Using blood cytokine measures to define high inflammatory biotype of schizophrenia and schizoaffective disorder. 2017;14(1):188.

75. Flatow J, Buckley P, Miller BJJBp. Meta-analysis of oxidative stress in schizophrenia. 2013;74(6):400409.

76. Fraguas D, Diaz-Caneja CM, Rodriguez-Quiroga A, Arango CJIJoN. Oxidative stress and inflammation in early onset first episode psychosis: a systematic review and meta-analysis. 2017;20(6):435-444.

77. Zhang M, Zhao Z, He L, Wan CJSCLS. A meta-analysis of oxidative stress markers in schizophrenia. 2010;53(1):112-124.

78. Eaton WW, Byrne M, Ewald H, et al. Association of schizophrenia and autoimmune diseases: linkage of Danish national registers. 2006;163(3):521-528.

79. Barron H, Hafizi S, Andreazza A, Mizrahi RJIjoms. Neuroinflammation and oxidative stress in psychosis and psychosis risk. 2017;18(3):651.

80. Miller BJ, Goldsmith DRJN. Towards an immunophenotype of schizophrenia: progress, potential mechanisms, and future directions. 2017;42(1):299.

81. Manu P, Correll CU, Wampers M, et al. Markers of inflammation in schizophrenia: association vs. causation. 2014;13(2):189-192.

82. Miller BJ, Buckley PFJPC. The case for adjunctive monoclonal antibody immunotherapy in schizophrenia. 2016;39(2):187-198.

83. Koga M, Serritella AV, Sawa A, Sedlak TW. Implications for reactive oxygen species in schizophrenia pathogenesis. Schizophrenia research. 2016;176(1):52-71.

84. Noto C, Ota VK, Gadelha A, et al. Oxidative stress in drug naive first episode psychosis and antioxidant effects of risperidone. Journal of psychiatric research. 2015;68:210-216.

85. Schiavone S, Trabace L. Pharmacological targeting of redox regulation systems as new therapeutic approach for psychiatric disorders: a literature overview. Pharmacological research.2016;107:195-204.

86. Miyake N, Miyamoto S, Yamashita Y, Ninomiya Y, Tenjin T, Yamaguchi N. Effects of N-acetylcysteine on cognitive functions in subjects with an at-risk mental state: a case series. Journal of clinical psychopharmacology. 2016;36(1):87-88.

87. Sarandol A, Sarandol E, Acikgoz HE, Eker SS, Akkaya C, Dirican M. First-episode psychosis is associated with oxidative stress: effects of short-term antipsychotic treatment. Psychiatry and clinical neurosciences. 2015;69(11):699-707.

88. Perkins DO, Jeffries CD, Addington J, et al. Towards a psychosis risk blood diagnostic for persons experiencing high-risk symptoms: preliminary results from the NAPLS project. Schizophrenia bulletin. 2015;41(2):419-428.

89. Miller BJ, Goldsmith DR. Towards an immunophenotype of schizophrenia: progress, potential mechanisms, and future directions.Neuropsychopharmacology. 2017;42(1):299-317.

90. Nunes S, Matsuo T, Kaminami MS, Watanabe M, Reiche E, Itano EN. An autoimmune or an inflammatory process in patients with schizophrenia, schizoaffective disorder, and in their biological relatives.Schizophrenia research. 2006;84(1):180.

91. Martinez-Gras I, Garcia-Sanchez F, Guaza C, et al. Altered immune function in unaffected first-degree biological relatives of schizophrenia patients. Psychiatry research.2012;200(2-3):1022-1025. 
92. Manu P, Correll CU, Wampers M, et al. Markers of inflammation in schizophrenia: association vs. causation. World Psychiatry.2014;13(2):189.

\section{Figure Legends}

\begin{tabular}{|c|c|c|c|c|c|}
\hline & & Control & Relative & Schizophrenia & p value \\
\hline \multirow{2}{*}{ Sex } & Female & 19 & 25 & 20 & NS \\
\hline & Male & 31 & 25 & 30 & NS \\
\hline \multicolumn{2}{|l|}{ Age } & $38,90 \pm 11,91$ & $45,22 \pm 15,44$ & $42,38 \pm 10,95$ & NS \\
\hline \multicolumn{2}{|c|}{ Education Level (year) } & $11.10 \pm 5.39^{*}$ & $9.81 \pm 4.82$ & $7.76 \pm 3.98$ & $<0.05$ \\
\hline \multirow[b]{2}{*}{ Job } & working & $36(\% 72)^{* *}$ & $26(\% 52)$ & $7(\% 14)$ & $<0.01$ \\
\hline & $\begin{array}{l}\text { not working / } \\
\text { student }\end{array}$ & $14(\% 28)$ & $24(\% 48)$ & $43(\% 86)^{* *}$ & $<0.01$ \\
\hline \multirow{2}{*}{$\begin{array}{l}\text { Marita } \\
\text { status }\end{array}$} & single & $21(\% 42)$ & $24(\% 48)$ & $41(\% 82)^{* *}$ & $<0.01$ \\
\hline & married & $29(\% 58)^{*}$ & $26(\% 52)^{*}$ & $9(\% 18)$ & $<0.05$ \\
\hline \multicolumn{2}{|c|}{ BMI } & $27,00 \pm 5,03$ & $26,51 \pm 5,22$ & $28,22 \pm 5,97$ & NS \\
\hline
\end{tabular}

Table 1. Demographic Characteristics of the Present Study, Data are presented as Mean \pm SD and the number of volunteers, ${ }^{*} \mathrm{p}<0.05,{ }^{* *} \mathrm{p}<0.01,{ }^{* * *} \mathrm{p}<0.001$ when compared with all groups.

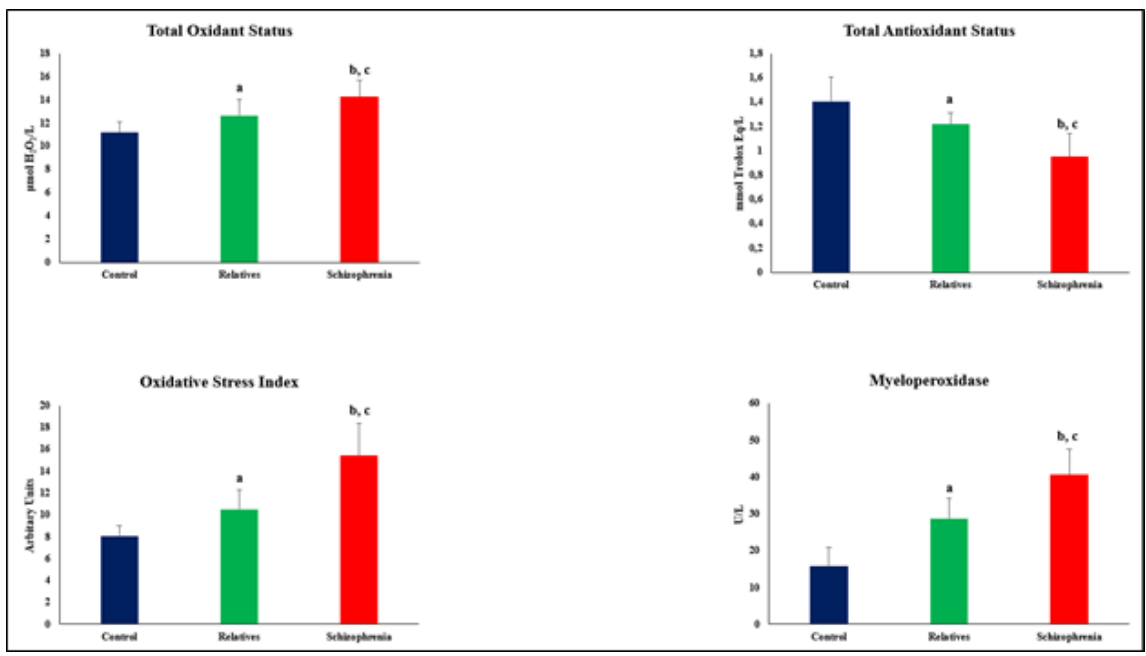

Figure 1. Changes of OS biomarkers levels of all groups. a - Statistically significant, compares control and relatives $(\mathrm{p}<0.05)$. b - Statistically significant, compares control and schizophrenia patients $(\mathrm{p}<0.05)$. c Statistically significant, compares relatives and schizophrenia patients $(\mathrm{p}<0.05)$. 


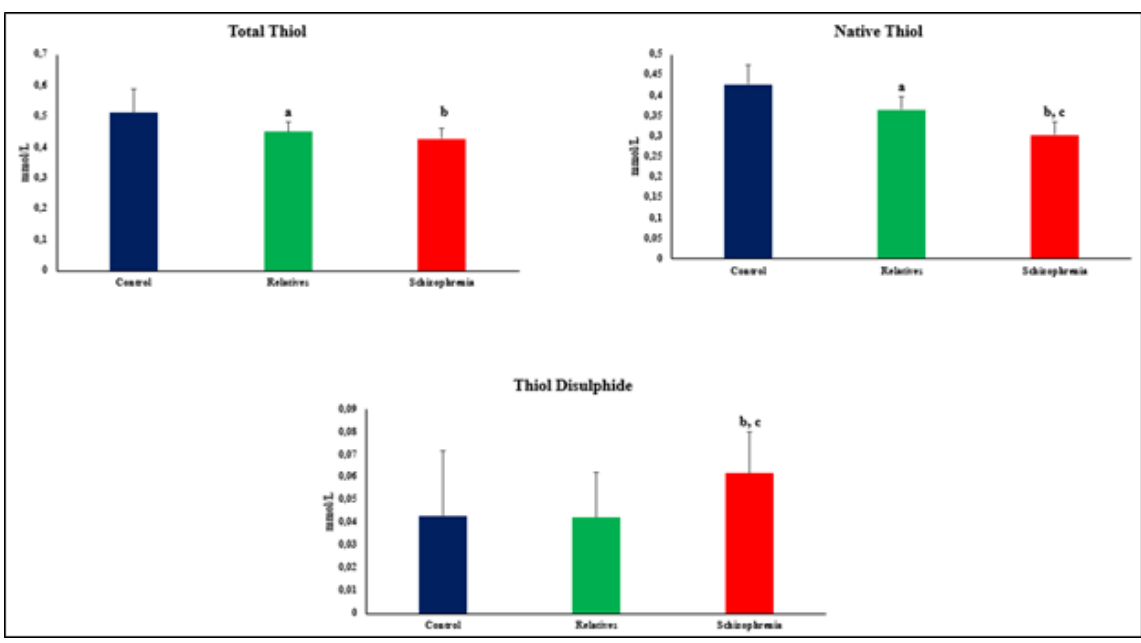

Figure 2. Changes of serum thiol-disulfide homeostasis biomarker levels of all groups. a - Statistically significant, compares control and relatives $(\mathrm{p}<0.05)$. b - Statistically significant, compares control and schizophrenia patients $(\mathrm{p}<0.05)$. $\mathrm{c}$ - Statistically significant, compares relatives and schizophrenia patients $(\mathrm{p}<0.05)$.

\begin{tabular}{|c|c|c|c|c|c|}
\hline & & Control & Relative & Schizophrenia & p value \\
\hline \multirow{2}{*}{ Sex } & Female & 19 & 25 & 20 & NS \\
\hline & Male & 31 & 25 & 30 & NS \\
\hline \multicolumn{2}{|l|}{ Age } & $38,90 \pm 11,91$ & $45,22 \pm 15,44$ & $42,38 \pm 10,95$ & NS \\
\hline \multicolumn{2}{|c|}{ Education Level (year) } & $11.10=5.39 *$ & $9.81 \pm 4.82$ & $7.76 \pm 3.98$ & $<0.05$ \\
\hline \multirow[b]{2}{*}{ Job } & working & $36(\% 72)^{* *}$ & $26(\% 52)$ & $7(\% 14)$ & $<0.01$ \\
\hline & $\begin{array}{l}\text { not working / } \\
\text { student }\end{array}$ & $14(\% 28)$ & $24(\% 48)$ & $43(\% 86)^{* *}$ & $<0.01$ \\
\hline \multirow{2}{*}{$\begin{array}{l}\text { Marita } \\
\text { status }\end{array}$} & single & $21(\% 42)$ & $24(\% 48)$ & $41(\% 82)^{* *}$ & $<0.01$ \\
\hline & married & $29(\% 58)^{*}$ & $26(\% 52)^{*}$ & $9(\% 18)$ & $<0.05$ \\
\hline BMI & & $27,00 \pm 5,03$ & $26,51 \pm 5,22$ & $28,22 \pm 5,97$ & NS \\
\hline
\end{tabular}

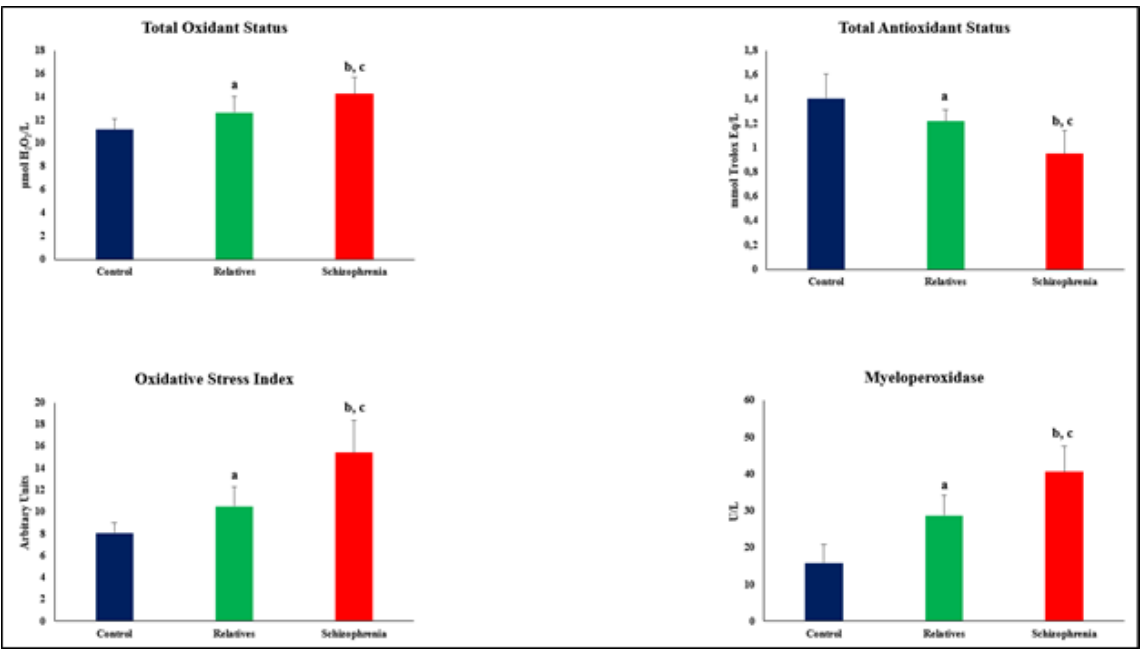




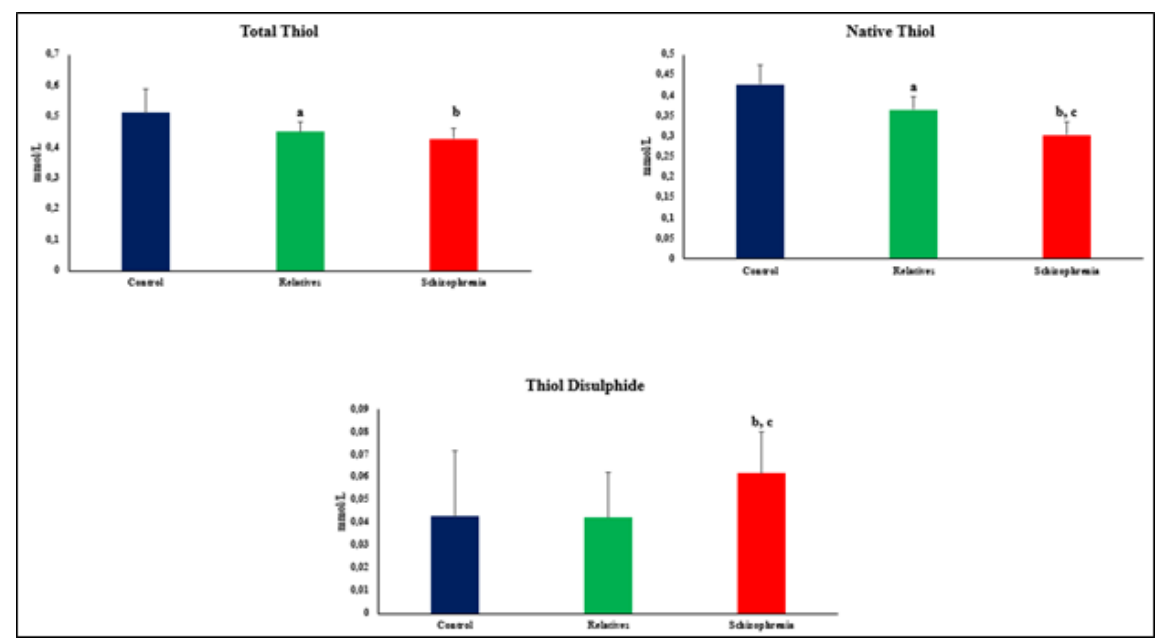

